\title{
Análisis de la cultura escolar en un Centro Preferente de Integración
}

\section{Ana Iglesias Rodríguez* María Isabel Calvo Álvarez** \\ *Escuela Universitaria de Educación de Ávila y Facultad de Educación; anaiglesias@usal.es **Universidad de Salamanca; isabelc@usal.es}

Recibido: 13 de octubre de 2010

\section{RESUMEN}

La experiencia que se presenta muestra la percepción que tiene el profesorado de un centro preferente de integración de la ciudad de Salamanca (España) sobre la cultura escolar, con este fin se utilizan algunos indicadores extraídos del Index for Inclusion elaborado por Tony Booth y Mel Ainscow en el año 2005, a partir del análisis de algunas de las dimensiones establecidas para llevar a cabo en la práctica los procesos de inclusión. Uno de los indicadores de Escuela Inclusiva expuesto en el Index de Inclusión (Booth y Ainscow, 2005) es la "Cultura Escolar". Se estudió dicho aspecto con base en la opinión recogida de docentes implicados en el proceso de enseñanza-aprendizaje, mediante la aplicación de un cuestionario elaborado a partir de la selección de algunos de los ítems contenidos en las siete subdimensiones denominadas "construir comunidad" y las seis subdimensiones de "valores inclusivos". El análisis de los datos servirá para la evaluación del Centro y la planificación de propuestas de mejora en la inclusión para que no sólo el profesorado, sino también las familias y el alumnado sean cada vez más partícipes en la construcción de una Escuela para Todos.

\section{PALABRAS CLAVE}

Indicadores, inclusión, escuela inclusiva, enseñanzaaprendizaje, escuela para todos, educación.
Aceptado: 11 de noviembre de 2010

processes. One of the features of an inclusive school, as defined in this index, is precisely the "school culture", which we examined and reflected upon after gathering the answers supplied by the teachers immersed in the learning-teaching continuum to a questionnaire containing a selection of items from both the seven sub-dimensions known as "building community" and the six sub-dimensions found under the category of "inclusive values". The analysis of the data will serve a twofold purpose, namely, the provision of a general assessment of the school centre and the proposal of specific measures conducive to the improvement of inclusive performance and discrete courses of action and practice that may engage the full participation of all parties -teachers, families, pupils and the community at large- involved in the construction of a School for All.

\section{KEY WORDS}

Indicators, inclusion, inclusive school, teachinglearning, school for all, education. 


\section{INTRODUCCIÓN}

Esta experiencia se enmarca dentro de la línea de investigación de "Educación Inclusi$\mathrm{va}^{\prime \prime}{ }^{1}$, realizada por un equipo de profesoras del Departamento de Didáctica, Organización y Métodos de Investigación de la Facultad de Educación de la Universidad de Salamanca, a su vez miembro del Instituto Universitario de Integración en la Comunidad (INICO), el cual desde hace varios años trabaja en el ámbito de la Inclusión Educativa.

El trabajo se llevó a cabo con maestros de un centro preferente de integración de la ciudad de Salamanca (España), quienes constituyen un grupo heterogéneo de profesionales con una amplia trayectoria educativa. En España, se denominan centros preferentes de integración a aquellos que cuentan con recursos más específicos ante necesidades concretas como son niños y niñas con deficiencias auditivas, visuales y/o motoras con el fin de facilitar la labor de los profesionales que atienden a los alumnos con necesidades específicas de apoyo educativo.

En los últimos años ha aumentado el interés por la inclusión de todas las personas desde un punto de vista social, político y educativo. Surge entonces la preocupación por conocer la realidad educativa de los centros.

Antes de acercarnos al objeto de este trabajo la inclusión educativa -, se realiza, aunque sea someramente, un recorrido por las fases educativas por las que han transitado los alumnos con discapacidad desde 1960 hasta nuestros días: la segregación, la integración y la inclusión, sin olvidar que,

"...las tres no siguen un orden cronológico, ni un desarrollo lineal y progresivo, de forma que una situación deja, en un momento determinado, paso a la otra. Si es cierto que, en primer lugar, se dan las situaciones de segregación y, posteriormente, las de integración e inclusión, también es cierto que en la actualidad conviven las tres situaciones en la práctica." (García Pastor, 2003: 13).
En la década de los 6o, surgió en Europa el movimiento de normalización, movimiento crítico liderado por padres de jóvenes con deficiencia mental y profesionales que se cuestionaban el papel de la educación especial segregada reivindicando el derecho a una vida lo más normalizada posible y la utilización de los servicios ordinarios de su comunidad. Esta idea o principio, llevado al ámbito educativo, trajo como consecuencia la integración escolar; la escuela ordinaria es el contexto escolar más normalizado y, por tanto, al que deben asistir también los niños con discapacidad. La integración escolar se presentó así como una alternativa a la educación especial segregada.

Sin embargo, la integración escolar también ha sido y es cuestionada ya que no ha satisfecho las expectativas ni personales ni sociales, dando paso a otra propuesta social y educativa más ambiciosa que es la inclusión, la cual reivindica el derecho a una educación de calidad en igualdad de oportunidades y en contextos normalizados, no sólo para los alumnos con discapacidad, sino para todos los alumnos, independientemente de su condición personal, social, cultural, etcétera. En definitiva, una Escuela para Todos.

La integración y la inclusión como movimientos escolares hay que enmarcarlos dentro de un contexto social y cultural que reivindica la igualdad de todos los ciudadanos y el reconocimiento de sus derechos a una vida normalizada y participativa donde no existan razones para su exclusión. Por tanto, responde a una cuestión de derechos humanos. Igual que en el ámbito social estos planteamientos exigen cambios de actitudes, acciones, mentalidades y voluntad política que se reflejen en normas, medidas y apoyos que faciliten el ejercicio de estos derechos. En el ámbito educativo también está presente esta exigencia. La nueva cultura escolar que supone la integración y la inclusión requiere no sólo cambios citados, sino también los organizativos y curriculares, avalados por la administración educativa. 
Los orígenes de la inclusión hay que buscarlos en las recomendaciones que desde distintos foros internacionales han ido surgiendo:

»1990, Conferencia Mundial sobre Educación para Todos: La satisfacción de las Necesidades Básicas de Aprendizaje. Jomtiem (Tailandia).

»1994, Conferencia Mundial sobre Necesidades Educativas Especiales. Acceso y calidad. Salamanca (España).

»2000, Conferencia de las Américas sobre Educación para Todos. Santo Domingo (República Dominicana).

»2009, Conferencia Mundial sobre Educación Inclusiva. Afrontando el Reto: Derechos, Retórica y Situación Actual. Volviendo a Salamanca. Salamanca (España).

La educación inclusiva propugna una forma de hacer y de pensar que va más allá de las prácticas derivadas de la filosofía de la integración.

En este momento, en España se cuenta con el marco legal adecuado para dar un impulso a la escuela inclusiva, a la denominada Escuela para Todos. Desde un punto de vista educativo y normativo, en España la actual Ley Orgánica de Educación - 2/2006, de 3 de mayo (LOE)- se justifica por la necesidad de mejorar la calidad de la educación que reciben los todos los jóvenes, sin exclusiones.

"... La atención a la diversidad se establece como principio fundamental que debe regir toda la enseñanza básica, con el objetivo de proporcionar a todo el alumnado una educación adecuada a sus características y necesidades." (p. 954).

A la exigencia de calidad se une la de equidad, entendida como la valoración de la individualidad personal y la aplicación de la igualdad teniendo en cuenta las diferencias entre personas y grupos para hacer una sociedad más justa; es decir, que la educación llegue a todos y garantice la igualdad efectiva de oportunidades, prestando los apoyos necesarios a los centros y a los alumnos.

"... Tres son los principios fundamentales que presiden esta Ley. El primero consiste en la exigencia de proporcionar una educación de calidad a todos los ciudadanos de ambos sexos, en todos los niveles del sistema educativo... Se trata de conseguir que todos los ciudadanos alcancen el máximo desarrollo posible de todas sus capacidades, individuales y sociales, intelectuales, culturales y emocionales para lo que necesitan recibir una educación de calidad adaptada a sus necesidades. Al mismo tiempo, se les debe garantizar una igualdad efectiva de oportunidades prestando los apoyos necesarios, tanto al alumnado que lo requiera como a los centros en los que están escolarizados. En suma, se trata de mejorar el nivel educativo de todo el alumnado, conciliando la calidad de la educación con la equidad de su reparto.

El segundo principio consiste en la necesidad de que todos los componentes de la comunidad educativa colaboren para conseguir ese objetivo tan ambicioso. La combinación de calidad y equidad que implica el principio anterior exige ineludiblemente la realización de un esfuerzo compartido... Pero la responsabilidad del éxito escolar de todo el alumnado no sólo recae sobre el alumnado individualmente considerado, sino también sobre sus familias, el profesorado, los centros docentes, las administraciones educativas, en última instancia, sobre la sociedad en su conjunto, responsable última de la calidad del sistema educativo. El principio del esfuerzo, que resulta indispensable para lograr una educación de calidad, debe aplicarse a todos los miembros de la comunidad educativa.

... El tercer principio consiste en un compromiso decidido con los objetivos educativos 
planteados por la Unión Europea para los próximos años..." (LOE: 951-952).

Esto no sería posible sin la colaboración de todos los integrantes de la comunidad educativa (equipo directivo, profesores, alumnos, padres y representantes del contexto social en su conjunto).

"... Cada uno de ellos tendrá que realizar una contribución específica. Las familias habrán de colaborar estrechamente y deberán comprometerse con el trabajo cotidiano de sus hijos y con la vida de los centros docentes. Los centros y el profesorado deberán esforzarse por construir entornos de aprendizaje ricos, motivadores y exigentes. Las administraciones educativas tendrán que facilitar a todos los componentes de la comunidad escolar el cumplimiento de sus funciones, proporcionándoles los recursos que necesitan y reclamándoles al mismo tiempo su compromiso y esfuerzo. La sociedad, en suma, habrá de apoyar al sistema educativo y crear un entorno favorable para la formación personal a lo largo de toda la vida. Solamente el compromiso y el esfuerzo compartido permitirán la consecución de objetivos tan ambiciosos" (LOE: 951).

Por tanto, lo que pretende la Ley Orgánica de Educación (LOE) en España es una educación de calidad para todos y entre todos, y este reto debe ser asumido no sólo por la administración educativa y la comunidad escolar, sino también por el conjunto de la sociedad. Es posible ver en el Preámbulo de esta ley la influencia de la filosofía de la escuela inclusiva.

"...La adecuada respuesta educativa a todos los alumnos se concibe a partir del principio de inclusión, entendiendo que únicamente de este modo se garantiza el desarrollo de todos, se favorece la equidad y se contribuye a una mayor cohesión social. La atención a la diversidad es una necesidad que abarca todas las etapas educativas y a todos los alumnos. Es decir, se trata de contemplar la diversidad de las alumnos y alumnas como principio y no como una medida que corresponde a las necesidades de unos pocos" (LOE: 956).

Por otra parte, a fin de garantizar la equidad, el Título II de la Ley Orgánica de Educación (LOE) denominado "Equidad en la Educación", cuando se refiere a la escolarización de alumnos que presentan necesidades específicas de apoyo educativo, contempla que ésta se regirá por los principios de normalización e inclusión. Es importante que se recoja dicho principio, pues desde nuestro punto de vista, es la primera vez que aparece en un texto legal como es la reciente Ley Orgánica de Educación promulgada en España en el año 2006. El Preámbulo reconoce que una adecuada atención a la diversidad debe partir del concepto de inclusión.

\section{- Planificación y desarrollo}

En la presente investigación se muestra la percepción que tiene el profesorado sobre la cultura escolar, se emplean algunos indicadores extraídos del Index for Inclusion (Booth y Ainscow, 2005). De las tres dimensiones que contempla el Index -A. Crear Culturas Inclusivas, B. Elaborar Políticas Inclusivas y C. Desarrollar Prácticas Inclusivas- fue seleccionada la primera para el desarrollo del trabajo, la cual, para Booth y Ainscow, 2005:

"...se centra en crear una comunidad segura, acogedora, colaboradora y estimulante en la que cada uno es valorado. Este hecho es el fundamento primordial para que todo el alumnado tenga los mayores niveles de logro. La finalidad es desarrollar valores inclusivos compartidos por todo el profesorado, el alumnado, los miembros del Consejo Escolar y las familias." (p. 163).

Esta dimensión tiene su correlato con la tarea de elaborar y hacer explícitos los proyectos educativos de los centros escolares, documentos 
institucionales donde se explicitan sus señas de identidad, sus metas, los valores que orientan su acción educativa y los principios básicos de organización escolar. Los principios que se derivan de esta cultura escolar son los que guían las decisiones que se concretarán en la segunda dimensión a la que hace referencia el Índice para la Inclusión.

-Elaborar Políticas Inclusivas- en cada centro. Éstas serán las encargadas de asegurar que los valores compartidos hacia la diversidad del alumnado sean el objetivo prioritario en las decisiones curriculares y organizativas necesarias para mejorar el aprendizaje y la participación de todos los estudiantes. Y, por último, será en la tercera dimensión "Desarrollar Prácticas Inclusivas" en el aula, donde se reflejarán la cultura y las decisiones colectivas adoptadas para sustentar la Educación Inclusiva.

Es importante subrayar que la cultura escolar es capaz de provocar cambios en las políticas y en las prácticas que pueden mantenerse y transmitirse a los nuevos miembros de la comunidad escolar. Con el fin de conocer la percepción que el profesorado del centro preferente de integración objeto de estudio tenía sobre la dimensión "Crear Culturas Inclusivas", se diseñó un cuestionario que denominamos "Cuestionario sobre Inclusión", teniendo muy en cuenta que el objetivo principal de este estudio es conocer la percepción del profesorado en torno a algunos de los indicadores de las dimensiones de la cultura escolar: construir comunidad y establecer valores inclusivos, contemplados en el documento de Booth y Ainscow.

El cuestionario fue diseñado y adaptado por un grupo de profesoras de Atención a la Diversidad de la Facultad de Educación de la Universidad de Salamanca, se tomaron como referente principal los indicadores establecidos en el "Index for Inclusion", elaborado por Tony Booth y Mel Ainscow en el año 2000 y actualizado en el año 2002. Los indicadores fueron publicados en el Reino Unido por el Centro de estudios para la Educación Inclusiva y adaptados al contexto español como Guía para la Evaluación y Mejora de la Educación Inclusiva (Sandoval, López, Miquel Durán, Giné y Echeíta, 2002). Se trata de una adaptación basada en la traducción al castellano realizada por la Oficina Regional de la UNESCO para América Latina y el Caribe (OREALC).

Previo a la selección definitiva, se reflexionó sobre el número y el contenido de los ítems a fin de adecuarlos a las características propias de las Etapas de Educación Infantil y Primaria y al tiempo de cumplimentación del mismo (que no fuera demasiado extenso). Quedó conformado por 50 ítems que se organizaron en dos secciones: los cinco primeros bloques se encuadran en la sección denominada Construir comunidad y los seis bloques siguientes forman parte de la segunda sección denominada Establecer valores inclusivos.

\section{- Contextualización del Centro}

El Colegio Público preferente de integración objeto de estudio es un centro que nació como una respuesta de la Obra Social de Caja Duero a las necesidades de escolarización que presentaba el barrio en el que se encuentra situado. Abrió sus puertas en septiembre de 1981 y en el curso 1985/86 se incorporó, como centro piloto, al Plan Experimental de Integración escolarizando alumnos con necesidades educativas especiales. Desde el curso escolar 1997/1998, acoge también en sus aulas a alumnos con dificultades motoras.

El colegio consta de tres unidades de educación infantil y seis unidades de educación primaria que atienden a 110 alumnos y alumnas, con una plantilla de 15 profesores y profesoras, 10 personas en administración y servicios y el apoyo del equipo de orientación. Hay 21 niños y niñas con necesidades educativas especiales de los cuales 17 son niños y niñas con dificultades motóricas y los cuatro restantes con discapacidad auditiva e intelectual.

El profesorado se distribuye en las siguientes especialidades: tres profesoras de Educación 
Infantil a tiempo completo, seis profesoras/ es tutores de Educación Infantil y Primaria, un especialista de Lengua Inglesa, un profesor de Educación Física, un profesor de Audición y Lenguaje, un profesor de Pedagogía Terapéutica a tiempo completo y dos más a tiempo parcial, un profesor de Música compartido con otro centro y un profesor de Religión. El equipo de Orientación Educativa y Psicopedagógica está formado por un psicopedagogo que asiste al centro un día a la semana y una trabajadora social que visita el centro cada quince días.

El cuestionario fue cumplimentado, voluntariamente, por aquellos profesores que quisieron participar en este estudio. En primer lugar, se llevó a cabo una reunión explicativa sobre la filosofía de la inclusión, se presentó el material de apoyo Index for Inclusion y los retos de su puesta en práctica para mejorar la organización y el funcionamiento del centro educativo. También se les informó sobre el contenido del cuestionario y los fines del mismo.

Una vez recogidos los cuestionarios, se procedió al análisis de cada uno de los ítems, para lo cual se creó una base de datos en el programa Excel de Microsoft.

A continuación, se presenta el estudio descriptivo de cada uno de los ítems que aparecen en el cuestionario, con el fin de proporcionar una visión global de la percepción que la población analizada mostró sobre el tema objeto de estudio.

\section{- Análisis de los datos}

El total de profesorado que trabaja en el Centro Preferente de Integración es de 13 profesores y profesoras, de los cuales nueve han respondido al cuestionario sobre inclusión; tres imparten su docencia en la Etapa de Educación Infantil, dos en la Etapa de Educación Primaria, dos son profesores de Educación Especial, uno de Logopedia/Audición y Lenguaje $y$, finalmente, otro realiza su tarea en Educación Infantil y Primaria. El intervalo de edad que engloba a más profesores es el comprendido entre los 50 y 65 años, destacando que la media de años de experiencia docente de los mismos es de 28 años.

Acorde con el número de ítems del cuestionario, se presentó una breve descripción de los resultados obtenidos por bloques de contenido. Se debe recordar que el cuestionario se centró en analizar la Dimensión Crear Culturas Inclusivas que a la vez estudia dos secciones: Construir $\mathrm{Co}$ munidad y Establecer Valores Inclusivos. A continuación se muestran los resultados obtenidos en cada una de ellas.

Los cinco primeros bloques se encuadran en la sección denominada Construir Comunidad.

El primero de ellos consta de 4 ítems que analizan el indicador "Todo el mundo merece sentirse acogido". Los resultados mostraron cómo todo el profesorado que participó en el estudio considera que el alumnado se siente identificado con su grupo de referencia. Asimismo, 8 de los 9 profesores opinaron que tanto el alumnado, como el profesorado y los miembros de la comunidad, sienten que forman parte del Centro; el profesor que no respondió a esta cuestión lo justificó diciendo que "hay ocasiones en la que sí se forma comunidad educativa, pero no siempre". Se destaca también que $50 \%$ del profesorado indicó que el centro sí tiene en cuenta las culturas locales y la diversidad cultural.

El segundo bloque ( 4 ítems) se centra en "La percepción de los profesores con respecto a si los alumnos se ayudan unos a otros". El profesorado opinó que el centro fomentaba el aprendizaje cooperativo entre sus alumnos, valorando de forma positiva el trabajo colaborativo en detrimento del trabajo individual. Con respecto a si los alumnos sienten que las disputas entre ellos son solucionadas justa y efectivamente, el profesorado consideró que sí; al igual ocurre con la percepción que el alumno tiene de los logros que cada uno de ellos pueden conseguir a lo largo de su etapa educativa.

El bloque tercero es el dedicado al indicador "Los profesores colaboran entre ellos". En este 
apartado, la mayoría de los profesores que participaron en el trabajo manifestaron que se tratan con respeto, trabajan de forma coordinada y colaborativa.

Para el cuarto apartado "El profesorado y el alumnado se tratan con respeto", los resultados señalan que el alumnado sabe a quién acudir cuando tiene un problema, que ellos se dirigen a los alumnos con respeto y tienen en cuenta su opinión sobre cómo introducir mejoras en el Centro. Igualmente señalaron que los alumnos creen que el profesorado lleva a cabo acciones eficaces para resolver sus dificultades.

El último módulo hace referencia a si "Existe colaboración entre el profesorado y las familias". Pues bien, $80 \%$ del profesorado considera que sí existe colaboración familia-escuela; los padres disponen de oportunidades para participar en el funcionamiento del centro, en el proceso educativo de sus hijos y, además, los docentes creen que sus preocupaciones son tenidas en cuenta.

Los seis bloques siguientes forman parte de la segunda sección denominada Establecer valores inclusivos. En las respuestas al ítem sobre si "Tienen expectativas altas sobre todo el alumnado", todos los profesores señalaron que en su quehacer diario valoran el logro del alumno en función de sus posibilidades, al igual que se les estimula para que su éxito dependa de su esfuerzo. De igual forma, $90 \%$ de la muestra respondió afirmativamente a los ítems formulados de la siguiente manera: se motiva a todo el alumnado a que tenga aspiraciones altas sobre su aprendizaje y se trata a todos ellos como si sus logros no tuvieran ningún tope. Finalmente, 70\% del profesorado evitó etiquetar a los discentes, especialmente a aquellos con dificultades de aprendizaje.

En el séptimo apartado "El profesorado, el alumnado y las familias comparten una filosofía de inclusión", los resultados obtenidos con respecto a la percepción que tienen los maestros del centro objeto de estudio sobre este aspecto es la siguiente: $60 \%$ señaló que construir comunidad escolar es tan importante como incre- mentar el rendimiento académico. En el mismo sentido, 50\% opinó que el trabajo colaborativo es tan importante como la motivación de la independencia o del trabajo individual. El 50\% de los profesores también consideró que la inclusión es un proceso de participación en todos los contextos.

El ítem "El profesorado piensa que todo el alumnado es igual de importante"; arrojó los siguientes resultados: $70 \%$ valora tanto al alumnado que tiene notas más bajas como al que las tiene muy altas; $60 \%$ ayuda al alumno para que valore su progreso personal independientemente de los resultados obtenidos. Por otra parte, $80 \%$ considera imprescindible la participación de la familia en el centro sin tener en cuenta el status laboral de las mismas; y $60 \%$ afirmó que las diferencias en las estructuras familiares son positivas.

El noveno bloque contenía el siguiente indicador "El profesorado y el alumnado son tratados como personas y como poseedores de un rol". Los datos obtenidos señalaron que la totalidad del profesorado afirma que proporciona al alumno un trato familiar $y / o$ afectivo y hacen de la tutoría un espacio donde él puede expresar sus preocupaciones, intereses, motivaciones y dudas sobre aspectos curriculares y personales; la razón es que consideran a todos como personas que aprenden y al mismo tiempo enseñan. Por último, se subraya que sólo $50 \%$ de los participantes en el estudio se siente apoyado y valorado por la comunidad educativa.

Las escasas y dispares respuestas obtenidas en el décimo bloque, referido a "El profesorado intenta eliminar todas las barreras al aprendizaje y la participación en el centro", indicaron una mala formulación del ítem y por consiguiente una dificultad en su comprensión.

Al analizar los datos del bloque "El centro se esfuerza en disminuir las prácticas discriminatorias", llamó la atención que uno de los profesores no contestara a ninguna de las preguntas formuladas ni realizara ninguna observación al respecto. Una gran mayoría (70\%) afirmó evitar 
las opiniones estereotipadas sobre la perfección física. Sin embargo, sólo $50 \%$ evita las actitudes estereotipadas hacia las personas con discapacidad. Teniendo en cuenta estas respuestas, destaca el hecho de que $60 \%$ del profesorado considera que la discapacidad se crea cuando las personas con deficiencias encuentran actitudes negativas y barreras institucionales.

\section{CONCLUSIONES}

Las sociedades actuales conceden una gran importancia a la educación que reciben los niños y los jóvenes pues de ellos depende tanto el bienestar individual como el colectivo.

Para la sociedad, la educación es el mejor medio de transmitir y de renovar la cultura y el conjunto de conocimientos y valores que la sustentan, de extraer las máximas posibilidades de sus fuentes de riqueza, de fomentar la convivencia democrática y el respeto a las diferencias individuales, de promover la solidaridad y evitar la discriminación, con el objetivo fundamental de lograr una cohesión social.

La preocupación existente por ofrecer una educación capaz de responder a las necesidades y demandas individuales y sociales no es nueva, así la concepción de la educación como un instrumento de mejora de la condición humana y de la vida colectiva ha sido una constante, aunque no siempre se hayan obtenido los resultados esperados.

Hoy día, todavía son muchos los que creen que llevan a cabo acciones verdaderamente inclusivas y no son conscientes de que siguen teniendo una concepción errónea e, incluso, una falta de información y de formación importante para poner en funcionamiento verdaderas prácticas inclusivas. El hecho de realizar actividades y/o proyectos relacionados con la atención a la diversidad, no implica que esas acciones vayan a convertir ni al centro ni al aula en inclusivos o en favorecedores de la esta práctica.
Por todo ello, es necesario reflexionar sobre las acciones implementadas en todos los ámbitos de la vida pero, sobre todo, en la escuela y en la repercusión de las mismas sobre los estudiantes, empezando - en primer lugar- por el profesorado.

El conocer la percepción que tiene el profesorado que trabaja en un centro preferente de integración en Salamanca (España) sobre la cultura escolar ha sido el cometido de este estudio. Del análisis de los datos obtenidos, concluimos lo siguiente:

1. Algunos profesores desarrollan prácticas que tienen que ver con la cultura y los valores de la inclusión. Entre las actividades que ponen en práctica se encuentran: fomentar el aprendizaje cooperativo mediante grupos de trabajo, tutoría entre iguales, aprendizaje recíproco, resolución de conflictos mediante el diálogo y el trabajo compartido entre profesores.

2. El Centro ha ido avanzando de las prácticas integradoras a otras que tienen que ver con la cultura inclusiva. Posiblemente, este avance se ha visto favorecido porque en los últimos años la ratio profesor-alumno ha descendido de forma notable en este Centro, no todos los centros tienen este aspecto favorecedor de las prácticas inclusivas.

3. Se detecta en el profesorado falta de contenidos teóricos, de conceptos y de terminología sobre la cultura de inclusión. El bloque número diez acusa más que ningún otro esas deficiencias y esta puede ser la causa de las escasas respuestas emitidas.

4. Algunos ítems del Index para la Inclusión ofrecen dificultad para su comprensión. Sería conveniente hacer una revisión de los mismos para formularlos más clara e inteligiblemente. 
Por último, si bien la muestra utilizada para este estudio hace referencia a un grupo concreto y reducido se cree, por los resultados obtenidos en el trabajo, que se puede iniciar un proceso de formación que ayude a reflexionar a los docentes sobre la cultura y los valores necesarios para lograr una inclusión educativa efectiva y real. El objetivo es que los centros inicien procesos de mejora para transformarse en centros inclusivos en dónde alumnos, profesores, familias y personal externo participen plenamente en su organización y desarrollo.

\section{NOTAS}

1. El grupo de investigación sobre Inclusión Educativa junto con profesores de la Universidad de Costa Rica y de la Universidad Estatal a Distancia de Costa Rica participa también en un Proyecto de Investigación subvencionado por la Agencia Española de Cooperación Internacional para el Desarrollo (AECID) sobre la misma temática.

\section{REFERENCIAS}

Booth, T. \& Ainscow, M. (2002, $2^{\text {aed. }}$.). Index for Inclusion. Developing learning and participation in schools. Mancherter: CSIE (Traducido al castellano de A.L. López, D. Durán, G. Echeita, C Giné, E. Miquel y M. Sandoval (2002). Guía para la evaluación y mejora de la educación inclusiva. Madrid: Consorcio Universitario para la Educación Inclusiva).

Booth, T. (2006). Manteniendo el futuro con vida; convirtiendo los valores de la inclusión en acciones En M.A. Verdugo y B. Jordán de Urríes (Coords). Rompiendo Inercias. Claves para avanzar. (pp. 211-217). Salamanca: Amarú.
Dyson, A. (2001). Dilemas. Contradicciones y variedades en la inclusión En M.A. Verdugo y $\mathrm{B}$. Jordán de Urries (coords.). Apoyo, autodeterminación y calidad de vida (pp. 145-16o). Salamanca: Amarú.

Echeita, G. (2006). Educación para la inclusión o educación sin exclusiones. Madrid: Narcea.

García Pastor, C. (2003). Segregación, Integración e Inclusión. Revista de Pedagogía, Vol. 55, 1, 9-26.

Illán, N. y Arnaiz, P. (1996). La evolución histórica de la Educación Especial. En N. Illán (Coord.). Didáctica y organización de la Educación Especial (pp. 13-43). Malága: Aljibe.

Ley Orgánica 2/2006, de 3 de mayo, de Educación (LOE). BOE (4 de mayo de 2006).

López, M. y Moreno, R. (2004). Reflexiones sobre la Declaración de Salamanca y la atención a las necesidades educativas especiales en la Comunidad Autónoma de Extremadura En G. Echeíta y M.A. Verdugo (Coords.). La Declaración de Salamanca sobre necesidades educativas especiales 10 años después. Valoración y Prospectiva (pp. 99-106). Salamanca: INICO.

Ortiz, C. (1995). Las personas con necesidades educativas especiales. Evolución histórica del concepto. En M.A. Verdugo (Coord.). Personas con discapacidad. Perspectivas psicopedagógicas y rehabilitadoras (pp. 37-77). Madrid: Siglo XXI.

UNESCO (2002). Declaración de Salamanca. Conferencia Mundial sobre Necesidades Educativas Especiales. Acceso y calidad. Madrid: UNESCO/ Ministerio de Educación y Ciencia.

VV.AA. (2006). Transformando la escuela: comunidades de aprendizaje. Barcelona: Graó. 
